\title{
INJECTIVE DE MORGAN AND KLEENE ALGEBRAS ${ }^{1}$
}

\section{ROBERTO CIGNOLI}

ABSTRACT. The aim of this paper is to characterize the injective objects in both the category of De Morgan algebras and the subcategory of Kleene algebras.

1. De Morgan and Kleene algebras. A De Morgan algebra $\langle\mathbf{A}, \wedge, \vee$, $\sim, 0,1>$ or, simply, $\mathbf{A}$, is an algebra with two binary $(\Lambda, \vee)$, one unary $(\sim)$ and two nullary $(0,1)$ operations satisfying the following axioms: $\langle\mathbf{A}, \wedge, \vee, 0,1\rangle$ is a distributive lattice with zero 0 and unit 1 , and:

$$
\text { (M1) } \sim \sim x=x \text { and }(\mathrm{M} 2) \sim(x \vee y)=\sim x \wedge \sim y .
$$

We shall consider only De Morgan algebras with more than one element.

These algebras have been studied by several authors under different names. Without the restriction of having a 0 and a 1 , they were introduced by G. Moisil [9] and further developed by J. Kalman [8] (who called them distributive i-lattices) and A. Bialynicki-Birula and H. Rasiowa [1], [2], [3] (under the name of quasi-Boolean algebras). We follow the terminology introduced by A. Monteiro [10], [11], that has become standard.

A De Morgan algebra $A$ such that the operation $\sim$ fulfills the condition:

$$
x \wedge \sim x \leq y \vee \sim y \quad \text { for any } x, y \text { in } \mathrm{A}
$$

is called a Kleene algebra (normal i-lattice in the terminology of [8]). Important examples of Kleene algebras are the Boolean algebras, the

Presented to the Society, December 10, 1973; received by the editors December $11,1973$.

AMS (MOS) subject classifications (1970). Primary 06A35, 02J99; Secondary $08 \mathrm{~A} 15$.

Key words and phrases. Distributive lattices, Boolean, De Morgan, Kleene, Lukasiewicz and Post algebras, injective algebras, retracts.

${ }^{1}$ This paperwas written while the author was a Visiting Fellow at the Department of Mathematics of Princeton University, supported by a fellowship from the Conse jo Nacional de Investigaciones de la República Argentina. 
Lukasiewicz and Post algebras (see, for instance, [4]) and the lattice ordered groups [8].

We shall denote by $\mathbf{T}$ the De Morgan algebra constructed as follows: as distributive lattice with 0 and 1 , take the Boolean algebra with two atoms $a$ and $b$, and define $\sim 0=1, \sim a=a, \sim b=b$ and $\sim 1=0$. ( $\mathbf{T}$ stands for "tetrada". We have borrowed this term from [11].) Moreover, $\mathbf{L}$ will denote the subalgebra of $\mathbf{T}$ with three elements and $\mathbf{0}$ the subalgebra of $\mathbf{T}$ with two elements.

Observe that $\mathbf{L}$ is a Kleene algebra and $\mathbf{0}$ a Boolean algebra.

Following A. Bialynicki-Birula and H. Rasiowa [2], for each prime filter $P$ of $\mathbf{A}$ (considered as a distributive lattice) we define the prime filter $g(P)=C \sim P$, where $C$ denotes the set-theoretical complement and $\sim P=$ $\{\sim x: x \in P\}$. The correspondence $P \mapsto g(P)$ plays an important role in the theory of representation of De Morgan algebras (see [2], [10], [13]). Here we are going to use it to recall some well-known facts about the $\mathbf{T}$-valued and L-valued homomorphisms that we shall need in what follows.

1.1. Proposition [1], [8], [11]. Let $\mathrm{A}$ be a De Morgan algebra and $h: \mathbf{A} \rightarrow \mathbf{T}$ a homomorphism. Then $P=h^{-1}(\{a, 1\})$ is a prime filter of $\mathbf{A}$ and $g(P)=h^{-1}(\{b, 1\})$. Conversely, if $P$ is a prime filter of $\mathbf{A}$, and if we de. fine

$$
f(x)= \begin{cases}1 & \text { if } x \in P \cap g(P), \\ a & \text { if } x \in P \backslash g(P), \\ b & \text { if } x \in g(P) \backslash P, \\ 0 & \text { if } x \notin \notin P \cup g(P),\end{cases}
$$

then $f: \mathbf{A} \rightarrow \mathbf{T}$ is a homomorphism and $f^{-1}(\{a, 1\})=P$, and $f^{-1}(\{b, 1\})=$ $g(P)$.

1.2. Proposition [1], [11]. Let $\mathbf{A}$ be a De Morgan algebra and $h: \mathbf{A} \rightarrow$ $\mathbf{L}$ a homomorphism. Then $P=b^{-1}\{(a, 1\})$ is a prime filter of $\mathbf{A}$ and $g(P)=$ $h^{-1}(\{1\})$. Conversely, let $P$ be a prime filter of $A$ such that $g(P) \subseteq P$ and define

$$
f(x)= \begin{cases}1 & \text { if } x \in g(P), \\ a & \text { if } x \in P \backslash g(P), \\ 0 & \text { if } x \notin \notin P .\end{cases}
$$

Then $f: \mathrm{A} \rightarrow \mathrm{L}$ is a homomorphism and $f^{-1}(\{a, 1\})=P$, and $f^{-1}(\{1\})=g(P)$. 
The only nontrivial subdirectly irreducible De Morgan algebras are $\mathbf{0}$, $\mathbf{L}$ and $\mathbf{T}$, and the only nontrivial subdirectly irreducible Kleene algebras are $\mathbf{0}$ and L [1], [8], [11]. For further reference, we are going to express a weaker form of these results in the form of a proposition. Recall first that if $\Lambda$ is a set and $\mathbf{A}$ a De Morgan algebra, then $\mathbf{A}^{\mathbf{\Lambda}}$ denotes the De Morgan algebra formed by all the functions from $\Lambda$ into $A$ with the operations defined pointwise.

1.3. Proposition. Given a De Morgan (Kleene) algebra A, there exists a nonempty set $\Lambda$ such that $\mathbf{A}$ is isomorphic to a subalgebra of $\mathbf{T}^{\mathbf{\Lambda}}\left(\mathbf{L}^{\mathbf{\Lambda}}\right)$.

Finally we wish to recall the following generalized De Morgan laws: If $\left\{x_{i}\right\}_{i \in I}$ is a family of elements of a De Morgan algebra A such that $\bigvee_{i \in I} x_{i}$ exists, then $\bigwedge_{i \in I} \sim x_{i}$ also exists and $\sim \bigvee_{i \in I} x_{i}=\bigwedge_{i \in I} \sim x_{i}$, and dually.

2. Centered De Morgan algebras. This section is the core of this paper. We shall begin by establishing some elementary relations between the operation $\sim$ and the Boolean complement. Then we introduce the notion of centered De Morgan algebra and deduce some of its properties that we shall need in the next section in order to characterize the injective De Morgan algebras.

If $\mathbf{A}$ is a distributive lattice with 0 and 1 , we shall denote the Boolean complement of an element $x$, if it exists, by $-x$, and the set of all complemented elements of $\mathbf{A}$ by $\mathbf{B}(\mathbf{A})$. It is well known that $\mathbf{B}(\mathbf{A})$ is a Boolean sublattice of A.

2.1. Lemma. The following conditions hold in any De Morgan algebra A:

(i) If $x \in \mathbf{B}(\mathbf{A})$, then $\sim x \in \mathbf{B}(\mathbf{A})$ and $-\sim x=\sim-x$.

(ii) If $c \in \mathbf{B}(\mathbf{A})$ and $c=\sim c$, then also $\sim-c=-c$.

(iii) If $\mathbf{K}(\mathbf{A})=\{x \in \mathbf{B}(\mathbf{A})$ : $-x=\sim x\}$, then $\mathbf{K}(\mathbf{A})$ is a subalgebra of $\mathbf{A}$ and a Boolean sublattice of $\mathbf{B}(\mathbf{A})$.

Moreoper, if $\mathbf{A}$ is complete, then $\mathbf{K}(\mathbf{A})$ is a complete sublatice of $\mathbf{B}(\mathbf{A})$.

Proof. (i) follows from the relations:

$$
\begin{aligned}
& \sim x \wedge \sim-x=\sim(x \vee-x)=\sim 1=0, \\
& \sim x \vee \sim-x=\sim(x \wedge-x)=\sim 0=1 .
\end{aligned}
$$

(ii) and (iii) are immediate consequences of (i). (Note that 0 and 1 always 
belong to $\mathbf{K}(\mathrm{A})$.) The last statement follows at once from the generalized De Morgan laws.

The next result will play an important role in what follows.

2.2. Lemma. Let $\mathbf{A}$ be a De Morgan algebra, $\mathbf{H}$ a Boolean sublattice of $\mathbf{K}(\mathrm{A})$, and suppose that $c$ is a complemented element of $\mathbf{A}$ such that $\sim c=c$. Then any element $x$ of the Boolean sublattice $\mathbf{S}(\mathbf{H}, c)$ generated by $\mathbf{H}$ and $c$ admits a unique representation of the form

$$
x=c \wedge k_{1} \vee-c \wedge k_{2} \text {, where } k_{1}, k_{2} \text { are in } \mathbf{H} \text {. }
$$

Proof. It is well known (see [14], or [6, p. 84]) that all the elements of $\mathbf{S}(\mathbf{H}, c)$ admit the representation (1). But, in general, this representation is not unique, and a necessary and sufficient condition in order that $\left(c \wedge k_{1}\right)$ $\vee\left(-c \wedge k_{2}\right)=\left(c \wedge k_{1}^{\prime}\right) \vee\left(-c \wedge k_{2}^{\prime}\right)$ is that the following two inequalities hold:

$$
c \leq\left(-k_{1} \vee k_{1}^{\prime}\right) \wedge\left(-k_{1}^{\prime} \vee k_{1}\right)
$$

and

$$
\left(k_{2} \wedge-k_{2}^{\prime}\right) \vee\left(k_{2}^{\prime} \wedge-k_{2}\right) \leq c .
$$

But if $c=\sim c$ and $k_{1}, k_{1}^{\prime}$ are in $\mathbf{H} \subseteq \mathbf{K}(\mathbf{A}),\left(1^{\prime}\right)$ is equivalent to

$$
-\left(\left(-k_{1} \vee k_{1}^{\prime}\right) \wedge\left(-k_{1}^{\prime} \vee k_{1}\right)\right)=\sim\left(\left(-k_{1} \vee k_{1}^{\prime}\right) \wedge\left(-k_{1}^{\prime} \vee k_{1}\right)\right) \leq \sim c=c \text {, }
$$

and this inequality together with ( $\left.1^{\prime}\right)$ imply

$$
\left(-k_{1} \vee-k_{1}^{\prime}\right) \wedge\left(-k_{1}^{\prime} \vee-k_{1}\right)=1
$$

which is equivalent to $k_{1}=k_{1}^{\prime}$. Analogously we can prove that ( $\left.1^{\prime \prime}\right)$ implies $k_{2}=k_{2}^{\prime}$.

2.3. Definition. A De Morgan algebra $A$ is called centered if there is an element $c$ (called a center of $\mathbf{A}$ ) such that $c \in \mathbf{B}(\mathbf{A}), c=\sim c$ and any $x$ in $\mathrm{A}$ can be written in the form:

$$
x=c \wedge k_{1} \vee-c \wedge k_{2} \text { with } k_{1}, k_{2} \text { in } \mathbf{K}(\mathbf{A})
$$

From the previous lemma it follows at once that for a given center $c$, the representation (C) is unique, and that $\mathbf{A}=\mathbf{B}(\mathbf{A})$, i.e., all its elements are complemented.

The following lemmas provide us with our main examples of centered De Morgan algebras. 
2.4. Lemma. For any nonempty set $\Lambda, \mathbf{T}^{\mathbf{\Lambda}}$ is a complete centered De Morgan algebra. Moreover, any element $c$ of $\mathbf{T} \boldsymbol{\Lambda}$, such that $c=\sim c$, is a center.

Proof. Suppose that $c=\left(c_{\lambda}\right)_{\lambda \in \boldsymbol{\Lambda}}$ is in $\mathbf{T}^{\boldsymbol{\Lambda}}$ and $c=\sim c$. This implies that $c_{\lambda}=\sim c_{\lambda}$ for all $\lambda \in \Lambda$, so $c_{\lambda}=a$ or $b$. If $x=\left(x_{\lambda}\right)$ is an element of $\mathbf{T}^{\Lambda}$, then for all $\lambda \in \Lambda$ we have $x_{\lambda}=k_{\lambda} \wedge c_{\lambda} \vee k_{\lambda}^{\prime} \Lambda_{-} c_{\lambda}$, where $k_{\lambda}$, $k_{\lambda}^{\prime}$ are 0 or 1 . Setting $k=\left(k_{\lambda}\right), k^{\prime}=\left(k_{\lambda}^{\prime}\right)$, we would have that $k, k^{\prime}$ are in $\mathbf{K}\left(\mathbf{T}^{\Lambda}\right)$ and that $x=c \wedge k \vee-c \wedge k^{\prime}$.

2.5. Lemma. The only finite centered De Morgan algebras are the products $\mathbf{T}^{k}, k$ integer $\geq 1$.

Proof. The above lemma implies that $\mathrm{T}^{k}$ is a (finite) centered De Morgan algebra for any integer $k \geq 1$. Conversely, let $\mathbf{A}$ be a finite centered De Morgan algebra. In particular $\mathbf{A}$ is a finite Boolean algebra. Denote by $\mathbf{M}$ the set of atoms of $\mathbf{A}$, and by $\mathbf{M}_{\boldsymbol{x}}$ the set of atoms preceding the element $x$. Since the prime filters of $A$ are exactly the filters generated by the atoms of $\mathbf{A}$, the Bialynicki-Birula and Rasiowa transformation between the prime filters of $\mathbf{A}$ introduced in $\$ 1$ induces a map $g: \mathbf{M} \rightarrow \mathbf{M}$ such that $g(g(m))=m$ for all $m$ in $\mathbf{M}$, and it is not hard to see that for any $x$ in $A$ we have

$$
\sim x=-\bigvee\left\{g(m): m \in \mathbf{M}_{x}\right\} .
$$

(A more general expression of $\sim$ in terms of the join-irreducible elements of any finite De Morgan algebra was obtained by A. Monteiro [10], [11].)

If $c=\sim c$, then it follows from (2) and from the fact that $c=\bigvee \mathbf{M}_{c}$, that $g(m) \neq m$ for any $m$ in $\mathbf{M}_{c}$. Since in this case we also have $-c=$ $\sim-c$ and $\mathbf{M}_{c} \cup \mathbf{M}_{-c}=\mathbf{M}$, we get that $g(m) \neq m$ for any $m$ in $\mathbf{M}$.

So we can define a map $g: \mathbf{M} \longrightarrow \mathbf{M}$ such that $g(g(m))=m$ and $g(m) \neq m$ for any $m$ in $\mathbf{M}$, and these conditions imply that $\mathbf{M}$ has an even number, $n$, of elements. Therefore, if $n=2 k$, we have that $\mathbf{A}=\mathbf{0}^{2 k}=\mathbf{T}^{k}$.

Remark. It is worthwhile to point out that if $\mathbf{A}$ is a finite Boolean algebra and $\mathbf{M}=\mathbf{M}(\mathbf{A})$ denotes the set of atoms of $\mathbf{A}$, then any function $g: \mathbf{M} \rightarrow \mathbf{M}$, such that $g(g(m))=m$ for all $m$, determines a structure of De Morgan algebra on $\mathbf{A}$ by means of the formula (2). Moreover, if we define $f(x)=-\sim x$, it follows from Lemma 2.1(i) that $f: \mathbf{A} \rightarrow \mathbf{A}$ is a Boolean algebra automorphism with the property that $f(f(x))=x$ for all $x$, and since $\sim m=-g(m)$ for any $m$ in $\mathbf{M}$, we see that $f$ is an extension of $g$. We can express these results, that are implicit in the mentioned works of A. Monteiro, in the following form: 
2.6. Proposition. Let $\mathrm{A}$ be a finite Boolean algebra. Then there is a one-to-one correspondence between the automorphisms $f: \mathrm{A} \rightarrow \mathbf{A}$ such that $f^{2}=$ identity on $\mathbf{A}$ and the maps $g: \mathbf{M}(\mathbf{A}) \rightarrow \mathbf{M}(\mathbf{A})$ such that $g^{2}=$ identity on $\mathrm{M}(\mathrm{A})$. In order that there exists such an automorphism $f$ with the property that $f(c)=-c$ for some $c$ in $\mathbf{A}$, it is necessary and sufficient that $\mathbf{A}$ have an even number of atoms.

Now we return to the study of some properties of centered De Morgan algebras that we shall need in the following section.

2.7. Lemma. Let $\mathbf{A}, \mathbf{A}^{\prime}$ be De Morgan algebras and $h: \mathbf{A} \rightarrow \mathbf{A}^{\prime}$ a homomorphism. Then the following properties hold:

(i) $h(\mathbf{B}(\mathbf{A})) \subseteq \mathbf{B}\left(\mathbf{A}^{\prime}\right)$, and $h(\mathbf{K}(\mathbf{A})) \subseteq \mathbf{K}\left(\mathbf{A}^{\prime}\right)$.

(ii) If $\mathbf{A}$ is centered, with a center $c$, and $h$ is onto, then $\mathbf{A}^{\prime}$ is also centered and $h(c)$ is a center.

Proof. Since a lattice homomorphism between bounded distributive lattices preserving 0 and 1 preserves the complement, it is plain that $h(\mathbf{B}(\mathbf{A})) \subseteq \mathbf{B}\left(\mathbf{A}^{\prime}\right)$, and since $h$ commutes with $\sim, h(\mathbf{K}(\mathbf{A})) \subseteq \mathbf{K}\left(\mathbf{A}^{\prime}\right)$. Suppose that $\mathbf{A}$ is centered and let $c$ be a center of $\mathbf{A}$. Then any $x$ in $\mathbf{A}$ can be written in the form (C), and $h(x)=b(c) \wedge h\left(k_{1}\right) \vee-h(c) \wedge h\left(k_{2}\right)$. Since by (i) $h\left(k_{1}\right), h\left(k_{2}\right)$ are in $\mathbf{K}\left(\mathbf{A}^{\prime}\right)$ and $h(c)=\sim h(c)$, it follows that $h(c)$ is a center of $h(\mathbf{A})$.

2.8. Theorem. Let A be a centered De Morgan algebra, C a complete De Morgan algebra and $\mathbf{S}$ a subalgebra of $\mathbf{A}$ containing a center $c$ of $\mathbf{A}$, and such that $c \wedge k_{1} \vee-c \wedge k_{2} \in \mathbf{S}$, with $k_{1}, k_{2}$ in $\mathbf{K}(\mathbf{A})$, implies that $k_{1}$, $k_{2}$ are in $\mathrm{S}$. Then any homomorphism $h: \mathrm{S} \rightarrow \mathrm{C}$ can be extended to a bomomorphism $f: \mathbf{A} \rightarrow \mathbf{C}$.

Proof. Let $h_{0}$ denote the restriction of $h$ to $\mathbf{K}(\mathbf{S})=\mathbf{S} \cap \mathbf{K}(\mathbf{A})$. K(S) is a Boolean sublattice of $\mathbf{K}(\mathrm{A})$, and since $h(\mathbf{K}(\mathbf{S})) \subseteq \mathbf{K}(\mathbf{C}), h_{0}$ is a Boolean homomorphism from the subalgebra $K(S)$ of the Boolean algebra $K(A)$ into the complete Boolean algebra $\mathbf{K}(\mathrm{C})$. By Sikorski's extension theorem [14], $\left[6\right.$, p. 142] there exists a Boolean homomorphism $f_{0}: K(A) \rightarrow K(C)$ extending $h_{0}$. Set $\mathbf{K}^{\prime}=f_{0}(\mathbf{K}(\mathbf{A}))$. $\mathbf{K}^{\prime}$ is a Boolean sublattice of $\mathbf{K}(\mathbf{C})$. Moreover, since $h$ preserves the complement, $h(c)$ is complemented in $\mathbf{C}$ and $-b(c)=$ $h(-c)$. If we define $f(x)=h(c) \wedge f_{0}\left(k_{1}\right) \vee-h(c) \wedge f_{0}\left(k_{2}\right)$, where $k_{1}, k_{2}$ are the elements of $\mathbf{K}(\mathrm{A})$ appearing in the representation $(\mathrm{C})$ of $x, f(x)$ will be an element of the Boolean lattice generated by $\mathbf{K}^{\prime}$ and $h(c)$. Since $h(c)=\sim h(c)$, from Lemma 2.2 it follows that $f_{0}\left(k_{1}\right)$ and $f_{0}\left(k_{2}\right)$ are uniquely 
determined, and from this property it follows at once that $f: \mathbf{A} \rightarrow \mathbf{C}$ is a homomorphism. Finally, if $s \in \mathrm{S}$, our hypothesis on $\mathbf{S}$ implies that the elements $c, k_{1}$ and $k_{2}$ appearing in the representation (C) of $s$ belong to $\mathrm{S}$, and then it is easy to check that $h(s)=f(s)$.

3. Injective De Morgan algebras. Recall that a De Morgan (Kleene) algebra $\mathbf{A}$ is said to be injective if, for any pair of De Morgan (Kleene) algebras $\mathbf{B}$ and $\mathbf{C}$, and one-to-one homomorphism $g: \mathbf{B} \rightarrow \mathbf{C}$, and any homomorphism $h: \mathbf{B} \rightarrow \mathbf{A}$, there exists a homomorphism $f: \mathbf{C} \rightarrow \mathbf{A}$ such that $f g=$ h. A De Morgan (Kleene) algebra $\mathbf{A}$ is a retract of the De Morgan (Kleene) algebra $\mathbf{B}$ if there are homomorphisms $h: \mathbf{A} \rightarrow \mathbf{B}$ and $f: \mathbf{B} \rightarrow \mathbf{A}$ such that $f h=$ identity on A.

Our first step will be to prove

3.1. Proposition. $\mathbf{T}$ is an injective De Morgan algebra.

Proof. It is enough to show that if $\mathrm{S}$ is a subalgebra of the De Morgan algebra $\mathbf{A}$, then any homomorphism $h: \mathbf{S} \rightarrow \mathbf{T}$ can be extended to a homomorphism $f: \mathbf{A} \rightarrow \mathbf{T}$. By Proposition $1.1, P^{\prime}=h^{-1}(\{a, 1\})$ is a prime filter of $\mathbf{S}$, and $g\left(P^{\prime}\right)=h^{-1}(\{b, 1\})$. Take a prime filter $P$ of $\mathrm{A}$ such that $P \cap S=P^{\prime}$ (the existence of such a $P$ is equivalent to the fact that the Boolean lattice 0 is injective in the category of distributive lattices). It is easy to check that $g(P) \cap S=g\left(P^{\prime}\right)$, and therefore the desired extension of $h$ is provided by the homomorphism $f: \mathrm{A} \rightarrow \mathrm{T}$ constructed in Proposition 1.1.

By taking into account that the direct product of injective algebras is injective, we get

3.2. Corollary. For any nonempty set $\Lambda, \mathbf{T}^{\mathbf{\Lambda}}$ is an injective De Morgan algebra.

From Proposition 1.3 and the above corollary follows

3.3. Corollary. Any De Morgan algebra can be embedded in an injective one.

Since the class of De Morgan algebras is equational, the last result implies that every De Morgan algebra has an injective hull.

3.4. Theorem. For a De Morgan algebra A the following conditions are equivalent:

(i) A is injective.

(ii) A is a retract of $\mathbf{T}^{\mathbf{\Lambda}}$, for some nonempty set $\Lambda$.

(iii) A is complete and centered. 
Proof. The equivalence between (i) and (ii) follows from Corollary 3.2 and Proposition 1.3 by using a standard technique.

(ii) implies (iii). It is well known that a retract of a complete Boolean lattice is a complete Boolean lattice [6, p. 146], and by Lemmas 2.7(ii) and 2.4, a retract of $\mathrm{T}^{\boldsymbol{\Lambda}}$ has to be a centered De Morgan algebra.

(iii) implies (ii). Let $\mathbf{A}$ be a complete centered De Morgan algebra. Then there exists a nonempty set $\Lambda$ and a one-to-one homomorphism $g$ : $A \rightarrow T^{\Lambda}$. Setting $\mathbf{S}=g(A)$, it is not hard to see that $S$ is a subalgebra of $\mathrm{T}^{\Lambda}$ fulfilling the conditions required in Theorem 2.8. Then if $h=g^{-1}$ : $\mathbf{S} \rightarrow \mathbf{A}$, by the same theorem there exists a homomorphism $f: \mathbf{T}^{\mathbf{\Lambda}} \rightarrow \mathbf{A}$ such that $f(g(x))=h(g(x))=x$ for all $x$ in A. So $\mathbf{A}$ is a retract of $\mathbf{T}^{\mathbf{\Lambda}}$.

From Lemma 2.5 and the above theorem we get

3.5. Corollary. The only finite injective. De Morgan algebras are the products $\mathrm{T}^{k}, k$ integer $\geq 1$.

Remark. From the above results it follows that the class of all injective De Morgan algebras is a proper subclass of the class of complete Boolean algebras. This shows the essential incorrectness of the main claims of [13] (see also [7]).

4. Injective Kleene algebras. The role played by the centered De Morgan algebras in the previous section will be played here by the centered three-valued Lukasiewicz algebras or Post algebras of order three. So we shall begin with a brief sketch of the properties of these algebras.

The following definition can be found in [5]. For further details and references, see [4].

4.1. Definition. A three-valued Lukasiewicz algebra is an algebra $\langle\mathbf{A}, \wedge, \vee, \sim, \nabla, 0,1\rangle$ such that $\langle\mathbf{A}, \wedge, \vee \sim, 0,1\rangle$ is a Kleene algebra and $\nabla$ is a unary operation on $\mathbf{A}$ fulfilling the conditions:

(L1) $\sim x \vee \nabla x=1$,

(L2) $x \wedge \sim x=\sim x \wedge \nabla x$,

(L3) $\nabla(x \wedge y) \leq \nabla x \Delta \nabla y$.

A three-valued Lukasiewicz algebra $\mathbf{A}$ is called centered if there is a (unique) element $c$ such that $\nabla c=1$ and $\sim \nabla \sim c=0$. The notion of centered three-valued Lukasiewicz algebra coincides with that of Post algebra of order three (see [4]).

The Kleene algebra $\mathbf{L}$ with the operation $\nabla$ defined by $\nabla a=\nabla 1=1$ and $\nabla 0=0$ is a centered three-valued Lukasiewicz algebra and so is $L^{\Lambda}$ for any nonempty set $\Lambda$. 
Since $\nabla x$ can be defined as the minimal element in $\mathbf{B}(\mathbf{A})$ that covers $x$ (see [5]), and since any De Morgan homomorphism preserves the complement, it follows that any De Morgan homomorphism between three-valued Lukasiewicz algebras also preserves the operation $\nabla$, i.e., is a Lukasiewicz homomorphism. Moreover, if $\mathbf{A}$ is a centered three-valued Lukasiewicz algebra, $\mathbf{A}^{\prime}$ a De Morgan algebra, and $h: \mathbf{A} \rightarrow \mathbf{A}^{\prime}$ an onto homomorphism, then $A^{\prime}$ is a centered three-valued Lukasiewicz algebra.

Finally we recall that the complete centered three-valued Lukasiewicz algebras are the injective objects in the category of three-valued Lukasiewicz algebras [12].

Now we are ready to establish the main facts about the injective Kleene algebras.

4.2. Proposition. $\mathbf{L}$ is an injective Kleene algebra.

Proof. Replace $\mathbf{T}$ by $\mathbf{L}$ in the proof of Proposition 3.1 and use Proposition 1.2.

4.3. Corollary. For any nonempty set $\Lambda, \mathrm{L}^{\Lambda}$ is an injective Kleene algebra.

4.4. Corollary. Any Kleene algebra can be embedded in an injective one.

The proof of the next theorem follows the same lines as that of Theorem 3.4 , by taking into account our remarks on centered three-valued Lukasiewicz algebras, and will be omitted.

4.5. Theorem. For a Kleene algebra A the following conditions are equivalent:

(i) A is injective,

(ii) $\mathbf{A}$ is a retract of $\mathbf{L}^{\Lambda}$ for some nonempty set $\Lambda$.

(iii) A is a complete centered three-valued Lukasiewicz algebra.

(iv) A is a complete Post algebra of order three.

\section{REFERENCES}

1. A. Bialynicki-Birula, Remarks on quasi-Boolean algebras, Bull. Acad. Polon. Sci. Cl. III 5 (1957), 615-619. MR 19, 629.

2. A. Bialynicki-Birula and H. Rasiowa, On the representation of quasi-Boolean algebras, Bull. Acad. Polon. Sci. Cl. III 5 (1957), 259-261. MR 19, 380.

3. - On constructible falsity in the constructive logic with strong negation, Colloq. Math. 6 (1958), 287-310. 
4. R. Cignoli, Moisil algebras, Notas de Lógica Matemática, no. 27, Instituto de Matemática, Univ. Nacional del Sur, Bahía Blanca, Argentina, 1970.

5. R. Cignoli and A. Monteiro, Boolean elements in Lukasiewicz algebras. II, Proc. Japan Acad. 41 (1965), 676-680. MR 34 \# 1238.

6. G. Gratzer, Lattice theory: First concepts and distributive lattices, Freeman, San Francisco, Calif., 1971.

7. J. S. Johnson, Review of [13], Zbl. Math. 248 (1973), \# 02068.

8. J. Kalman, Lattices with involution, Trans. Amer. Math. Soc. 87 (1958), 485-491. MR $20 \# 1641$.

9. G. Moisil, Recherches sur l'algèbre de la logique, Ann. Sci. Univ. Jassy 22 (1935), 1-117.

10. A. Monteiro, Matrices de Morgan caractéristiques pour le calcul propositionnel classique, An. Acad. Brasil. Ci. 32 (1960), 1-7. MR 28 \#18.

11. Lectures given at the Univ. Nacional del Sur, Bahía Blanca, Argentina, 1962.

12. L. Monteiro, Sur les algèbres de Lukasiewicz injectives, Proc. Japan Acad. 41 (1965), 578-581. MR 33 \#5536.

13. I. Petrescu, Injective objects in the category of Morgan algebras, Rev. Roumaine Math. Pures Appl. 16 (1971), 921-926. MR 44 \#3940.

14. R. Sikorski, A theorem on extension of homomorphisms, Ann. Soc. Polon. Math. 21 (1948), 332-335 (1949). MR 11, 76.

DEPARTAMENTO DE MATEMÁTICA, UNIVERSIDAD NACIONAL DEL SUR, BAHÍA BLANCA, ARGENTINA

Current address: Department of Mathematics, University of Illinois at Chicago Circle, Chicago, Illinois 60680 